\title{
Exogenous Lipoid Pneumonia: Dramatic Clinical and Radiological Improvement After Multiple Segmental Bronchoalveolar Lavages
}

\author{
Mohammadreza Modaresi, ${ }^{1}$ Minoo Dadkhah,, ${ }^{2, *}$ and Sayed Javad Sayedi ${ }^{1}$ \\ ${ }_{1}^{1}$ Division of Pediatrics Pulmonary Disease, Children's Medical Center, Pediatrics Center of Excellence, Tehran University of Medical Sciences, Tehran, IR Iran \\ ${ }^{2}$ Department of Pediatrics, Children's Medical Center, Tehran University of Medical Sciences, Tehran, IR Iran \\ ${ }^{*}$ Corresponding author: Minoo Dadkhah, Department of Pediatrics, Children's Medical Center, Tehran University of Medical Sciences, Tehran, IR Iran. Tel: +98-9124990059, \\ E-mail: minoodadkhah@yahoo.com
}

Received 2015 July 12; Accepted 2015 August 12

Keywords: Lipoid Pneumonia, Bronchoalveolar Lavage, Computed Tomography, Mineral Oil

\section{Dear Editor,}

Hydrocarbon compounds such as petroleum jelly and mineral oil can cause lower respiratory disease named exogenous lipoid pneumonia because of high viscosity and surface tension (1).

Acute exogenous lipoid pneumonia occurs after exposure to large amounts of mineral oils. The acute form in children is mostly a consequence of accidental poisoning. (2). Consolidation, nodular lesions, reticular and alveolar-interstitial patterns are the most common findings in the chest X-rays. Lower lobes or right middle lobe commonly get involved, but multifocal and bilateral lesions are shown too (3). The diagnosis of exogenous lipid pneumonia is made by a positive history of mineral oil exposure and radiologic findings in favor of the disease and revealing lipid-laden macrophages on bronchoalveolar lavages (BALs) or sputum specimen. Multiple BALs can remove Lipid-laden macrophages as a leading cause of fibrosis in the alveoli and interstitium $(3,4)$.

We present a rare case of exogenous lipoid pneumonia in a 2.5-year old boy with a history of mineral oil aspiration who admitted to the pediatric intensive care unit because of cyanosis and severe respiratory distress. On physical examination he presented with tachycardia (190 min), tachypnea (80 min), $\mathrm{O}_{2}$ saturation $50 \%$, numerous crackles on auscultation of both lungs. Laboratory findings were leukocytosis with a left shift and increased CRP. A chest X-ray showed diffuse opacities with an alveolar pattern in both lungs (Figure 1). CT scans of the chest revealed bilateral diffuse severe pneumonitis with ground glass and alveolar appearance and crazy paving (Figure 2). Bronchoscopy was done. Mucopurulent secretions aspirated then rinsed with saline. After staining the BAL fluid with Sudan III many lipid laden macrophages (grade IV/IV) were seen. Multiple segmental BAL was done weekly, after that BAL fluid became nearly clear and cell counts reached near the normal range values. CT scan which was done after the forth therapeutic BAL, showed dramatic improvement in both lungs (Figure 3). The patient discharged after one month hospitalization and recommended to return to follow up. The best way to remove the oil is multiple BALs especially in the most severely involved segments (3). According to several surveys, multiple BALs is an effective therapeutic strategy with few risks, and better improvement in the clinical and radiological signs (5). The present study indicates that therapeutic multiple segmental BAL is a successful method in removing intra-alveolar mineral oil with significant resolution of clinical, radiological and laboratory findings.

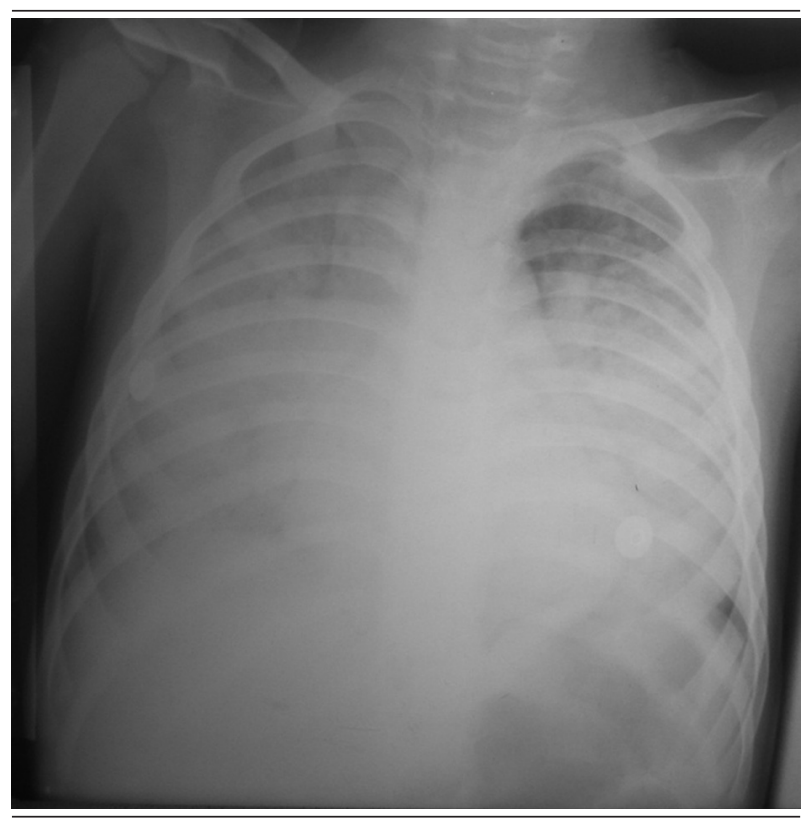

Figure 1. Simple Anteroposterior Chest X-Ray Demonstrating Bilateral Opacities, Mostly in the Lower Lobes

Copyright (C) 2015, Growth \& Development Research Center. This is an open-access article distributed under the terms of the Creative Commons Attribution-NonCommercial 4.0 International License (http://creativecommons.org/licenses/by-nc/4.0/) which permits copy and redistribute the material just in noncommercial usages, provided the original work is properly cited. 


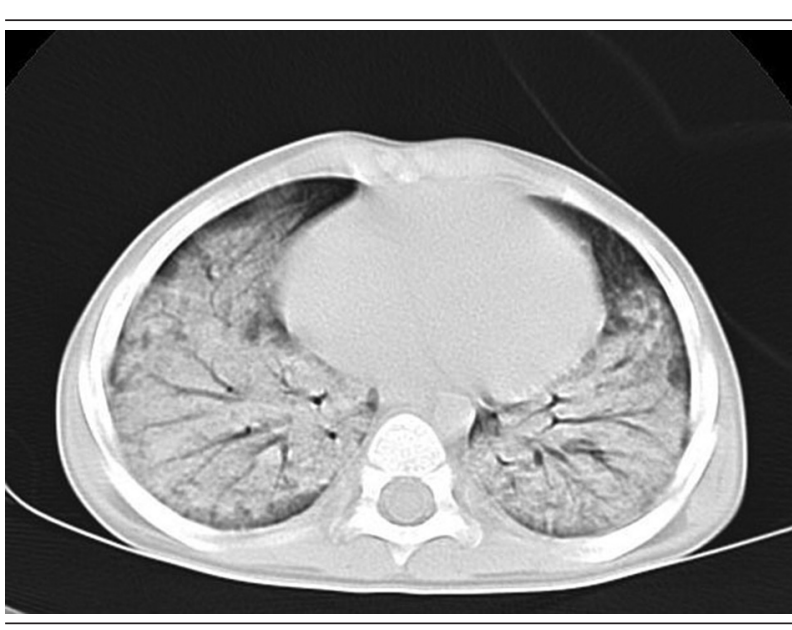

Figure 2. High-Resolution Computed Tomography Scan of the Chest Demonstrating Alveolar Infiltrate, Mostly in the Lung Bases

\section{References}

1. Mylonaki E, Voutsas V, Antoniou D, Papakosta D, Kontakiotis T Skordalaki A, et al. Hydrocarbon pneumonitis following liquid paraffin aspiration during a fire-eating performance: a case report. J Med Case Rep. 2008;2:214. doi: 10.1186/1752-1947-2-214. [PubMed: 18565234]

2. Pielaszkiewicz-Wydra M, Homola-Piekarska B, Szczesniak E, Ciolek-Zdun M, Fall A. Exogenous lipoid pneumonia - a case report of a fire-eater. PolJ Radiol. 2012;77(4):60-4. [PubMed: 23269939]

3. Sias SM, Daltro PA, Marchiori E, Ferreira AS, Caetano RL, Silva CS, et al. Clinic and radiological improvement of lipoid pneumo-

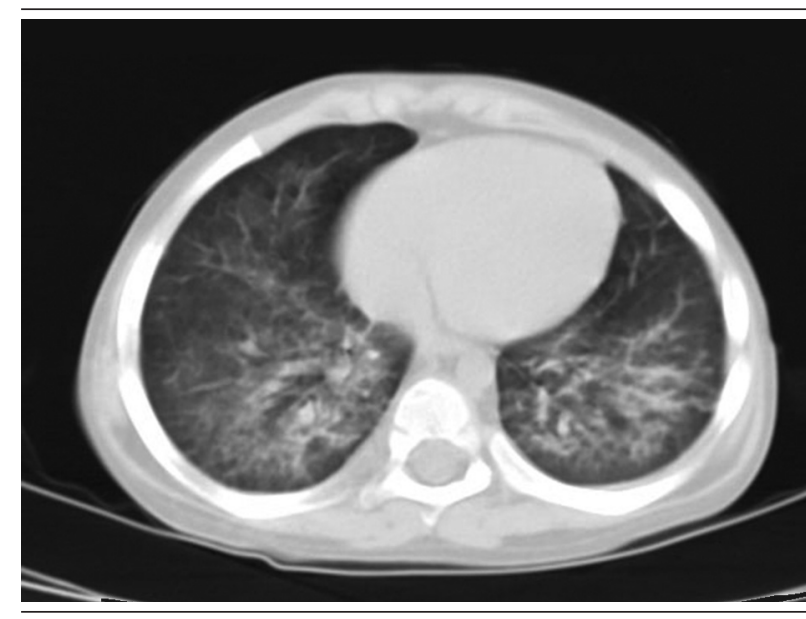

Figure 3. High-Resolution Computed Tomography Scan of the Chest Showing Dramatic Improvement in Both Lungs After Multiple Segmental BAL

nia with multiple bronchoalveolar lavages. Pediatr Pulmonol. 2009;44(4):309-15. doi: 10.1002/ppul.20918. [PubMed:19283836]

4. Marchiori E, Zanetti G, Mano CM, Hochhegger B. Exogenous lipoid pneumonia. Clinical and radiological manifestations. Respir Med. 2011;105(5):659-66. doi: 10.1016/j.rmed.2010.12.001. [PubMed: 21185165]

5. Simoes MC, Toro IF, Ribeiro JD, Toro AA. Lipoid pneumonia in a 40-day-old infant. J Bras Pneumol. 2012;38(4):535-7. [PubMed: 22964941] 